\title{
Diagnostica della calcolosi renale
}

\author{
G. Gambaro, B. Baggio \\ Istituto di Medicina Interna, Divisione di Nefrologia 1, Policlinico, Università di Padova
}

$\mathbf{L}$

a nefrolitiasi calcica ha assunto negli ultimi anni le caratteristiche di una vera malattia sociale: il $3 \%$ della popolazione mondiale $(12 \%$ negli USA) è destinato a formare almeno un calcolo nell'arco della vita. Come causa di ospedalizzazione la calcolosi renale è ai primi posti tra le patologie uronefrologiche. Infatti, fino al $20-30 \%$ dei nefrolitiasici necessita di un ricovero ospedaliero e di questi anche il $20 \%$ può richiedere indagini urologiche strumentali ed interventi chirurgici. La malattia inoltre, specie in alcune sue forme, è gravata da un elevato rischio di complicanze tra le quali anche l'evoluzione verso l'insufficienza renale cronica. Per la rilevanza sociale ed economica del problema si comprende l'importanza di una prevenzione primaria e secondaria della malattia. Queste ragioni hanno suscitato l'interesse di urologi e nefrologi per questa malattia, interesse che ha portato ad una maggiore comprensione della sua patogenesi, ad un più razionale approccio diagnostico ed alla possibilità di una adeguata profilassi farmacologica delle recidive.

Ci si può chiedere oggi se i progressi conseguiti dall'urologia endoscopica nella rimozione dei calcoli, nonché l'introduzione della litotripsia extracorporea ad onde d'urto (ESWL), che hanno rivoluzionato l'approccio terapeutico alla calcolosi urinaria, non abbiano reso pleonastico qualsiasi approccio interni- stico, diagnostico e profilattico/terapeutico. Nella Consensus Conference tenutasi nel 1988 al NIH Bethesda, Pak (1) elencava sette giustificazioni generali all'approccio internistico a questa patologia, giustificazioni che potrebbero essere facilmente riassunte in una: l'approccio medico può prevenire le recidive della calcolosi, mentre ciò non è possibile con il chirurgico.

\section{Iter diagnostico}

La calcolosi renale è la tappa comune finale di patologie diverse renali ed extrarenali. Essa può essere perciò malattia di per se stessa (nefrolitiasi idiopatica) o sintomo di malattia (Tab. I).

Il primo obiettivo è perciò quello di escludere tutte le possibili forme secondarie di nefrolitiasi, l'individuazione delle quali ha importanti conseguenze sia in terapia che per quanto riguarda la prognosi.

Sono stati proposti vari protocolli diagnostici per identificare le molteplici cause di nefrolitiasi (2-4). Protocolli estesi sono stati suggeriti nei casi di nefrolitiasi particolarmente severe, mentre nei soggetti al loro primo episodio nefrolitiasico gli iter diagnostici suggeriti sono assai più "compatti".

Mentre c'è accordo pressoché unanime sull'iter diagnostico nei calcolotici recidivanti ed in alcune particolari forme di nefrolitiasi, controverso è l'atteggiamento da seguire nei pazienti al loro primo episodio (3). Alcuni hanno proposto iter diagnostici approfonditi e costosi, dai

TAB. I - CLASSIFICAZIONE

\section{Nefrolitiasi idiopatica}

Nefrolitiasi secondaria

$\triangleright$ Renale

Tubulopatie:

Acidosi tubulare distale conclamata

Cistinuria

Nefropatie malformative:

Rene a spugna

Rene policistico

$\triangleright$ Anomalie delle vie urinarie

$\triangleright \quad$ Infezioni delle vie urinarie da germi ureasi-produttori

$\triangleright$ Extrarenale

Iperparatiroidismo primitivo

Sarcoidosi

Mieloma multiplo

Ossalosi tipo I e tipo II

Iperuricemia

Immobilizzazione prolungata

Intossicazione da vitamina D

Assunzione di farmaci litogeni

Enteropatie croniche

Bypass digiuno-ileale 
quali poi derivare approcci terapeutici personalizzati (9) considerando che: a) nei calcolotici singoli la frequenza di recidiva è molto alta $(70-80 \%$ a 20 anni) $(5,6)$; b) che la probabilità di individuare anomalie quali ad esempio l'ipercalciuria o l'iperuricuria si afferma sia essenzialmente simile a quella dei calcolotici recidivanti $(7,8)$; c) che l'effetto farmacologico preventivo sarebbe elevato (3).

Tuttavia un approccio diagnostico più semplice, che è quello generalmente adottato nel nostro centro, è giustificato se consideriamo che: d) la definizione di recidivanza è di scarsissimo aiuto nel contesto dei lavori portati ad esempio (5, 6) (probabilmente un paziente che recidiva dopo 5-10-15 anni dal precedente episodio dovrebbe essere considerato non un calcolotico recidivante, ma un nefrolitiasico occásionale o latente; e) che proprio nei lavori citati $(7,8)$ nei pazien-

\section{TAB. II - ANAMNESI}

Familiarità

per nefrolitiasi

per nefropatie

per malattie metaboliche

(iperuricemia/gotta, cistinuria,

ossalosi, poliendocrinopatie)

Abitudini di vita

dieta (abusi alimentari)

introito idrico

attività fisica (sudorazione profusa)

Farmaci litogeni

triamterene, acetazolamide

vitamina $C$, vitamina $D$

uricosurici, citostatici

Nefropatie malformative

Anomalie delle vie urinarie

Infezioni delle vie urinarie

Dietesi uratica

Osteopatie, fratture patologiche

Immobilizzazione prolungata

Malattie gastrointestinali croniche malassorbimento

ulcera peptica

bypass digiuno-ileale ti al primo episodio di calcolosi l'incidenza di iperparatiroidismo era notevolmente inferiore rispetto ai casi recidivanti, mentre era maggiore quella dei pazienti senza anomalie metaboliche identificabili; f) che l'affermazione della notevole efficacia profilattica del trattamento farmacologico sin dal primo episodio di calcolosi è molto discutibile perché mai verificata; ed infine g) che una larga percentuale di calcolotici trae beneficio da norme igienico-dietetiche del tutto generali (il cosiddetto "stone clinic effect") (10).

\section{Anamnesi ed esame obiettivo}

La valutazione diagnostica della nefrolitiasi è essenzialmente laboratoristica. Tuttavia l'anamnesi permette di ricavare alcune indicazioni sull'eziologia della malattia (Tab. II) e di precisarne 1"'attività", concetto questo essenziale per future decisioni terapeutiche.

Gli elementi da raccogliere a tal fine sono la recidivanza della calcolosi e gli intervalli tra le recidive, la notizia di precedenti interventi chirurgici per l'insorgenza di una complicanza ostruttiva, nonché di altre complicanze quali la pielonefrite, e il deterioramento della funzione renale. Estremamente modeste infine sono le indicazioni che si possono trarre dall'esame obiettivo.

\section{TAB. III - REPERTI RADIOLOGICI}

Calcolo radiotrasparente acido urico

Calcolo radioopaco a stampo: fosfato-ammoniomagnesiaco grande: cistina, fosfato di calcio piccolo: ossalato di calcio

Nefropatie malformative rene a spugna rene policistico

Anomalie primitive delle vie escretrici Complicanze ostruttive

Pielonefrite

Nefrocalcinosi

\section{Valutazione radiologica}

Tra le varie indagini radiologiche a cui tutti i pazienti calcolotici debbono essere sottoposti, l'urografia rimane ancora l'indagine cardine.

I reperti radiologici (Tab. III), oltre che chiarire la presenza di uno o più calcoli $o$ di altre patologie renali e delle vie escretrici, possono fronire una prima indicazione sul tipo di calcolosi e sulla sua primitività o secondarietà.

Per quanto riguarda in particolare la nefrocalcinosi, che talvolta viene occasionalmente osservata nel corso di indagini radiologiche eseguite per altri motivi, essa può essere un indizio di iperparatiroidismo primitivo, di rene con midollare a spugna, di sarcoidosi e di intossicazione da vitamina $D$.

\section{Valutazione di laboratorio}

Lo screening laboratoristico di un paziente nefrolitiasico non può ovviamente prescindere dai più comuni test di funzionalità renale. La tappa fondamentale della valutazione iniziale è l'analisi chimica del calcolo, purtroppo non sempre possibile, che permette di orientare il successivo iter diagnostico e terapeutico (Fig. 1).

Per quanto riguarda la calolosi calcica, che rappresenta oltre 1'80\% di tutte le forme di nefrolitiasi, da vari Autori sono stati proposti iter diagnostici differenziati, tendenti in particolare a chiarire l'origine dell'ipercalciuria e dell'iperossaluria idiopatiche che sono di frequente riscontro in questa malattia, nonché la valutazione di un deficit di attività inibitoria (escrezione urinaria di citrato, magnesio, pirofosfato, glicosaminoglicani). La complessità e il costo economico di un simile approccio diagnostico, possibile solo in pochi centri specializzati, a nostro parere non si traducono in un reale vantaggio per il paziente calcolotico ai fini terapeutici. Particolare attenzione va invece posta all'esclusione di un iperparatiroidismo primitivo, condizione questa molto più frequente di quanto si pensi e talora di difficile diagnosi in quanto $\mathrm{i}$ segni biochimici e clinici classici sono assenti o assai sfumati. Da sottolineare poi la necessità, in presenza di calcolosi 
Analisi chimica del calcolo

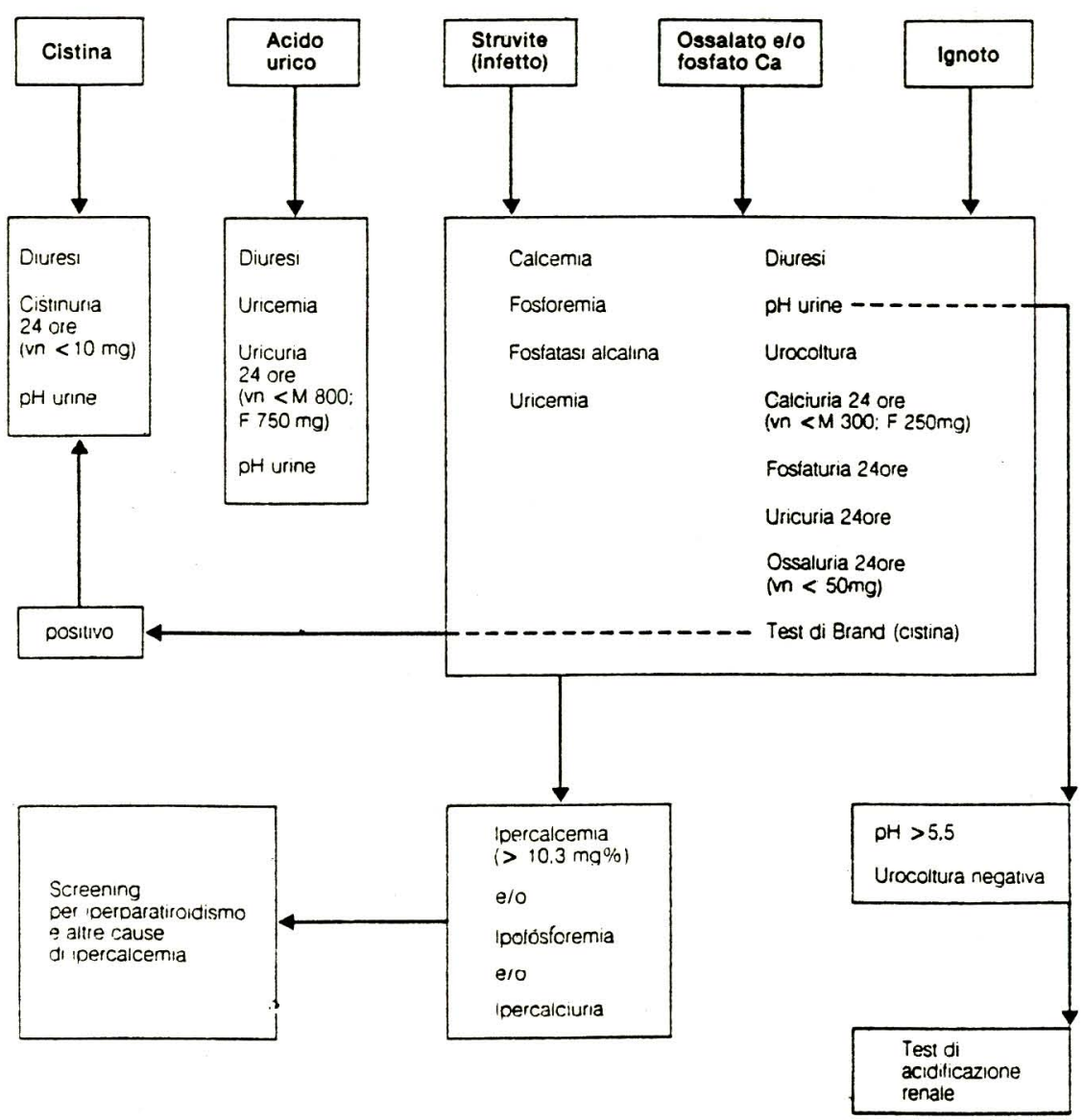

Fig. 1 - Analisi chimica del calcolo.

cistinica o di cistinuria, di estendere la valutazione laboratoristica e radiologica nei consanguinei.

Particolari problemi diagnostici sorgono in quei pazienti nei quali, pur in presenza di un quadro clinico suggestivo di nefrolitiasi (coliche renoureterali recidivanti e familiarità), i reperti umorali e radiologici non chiariscono la presenza di malattia litiasica (urografia normale, non espulsione di calcoli o renella, macro/microematuria). Questi soggetti, che comunque dovrebbero essere sottoposti allo screening laboratoristico per la calcolosi, potrebbero trarre vantaggio da test sensibili e specifici per la nefrolitiasi calcica. A tale uso potrebbe prestarsi il test da noi proposto, che permette di evidenziare un abnorme flusso transmem- brana eritrocitaria dell'ossalato nei pazienti calcolotici (11), anomalia questa che sembra avere un ruolo patogenetico nella calcolosi calcica idiopatica e che si è rivelata capace di discriminare $\mathrm{i}$ pazienti a rischio di recidiva (12).

\section{BIBLIOGRAFIA}

1. Pak CYC. Role of medical prevention. J Urol 1989; 141: 798.

2. Pak CYC, Britton F, Peterson $\mathrm{R}$, et al. Ambulatory evaluation of nephrolithiasis. Classification, clinical presentation and diagnostic criteria. Am J Med 1980; 69: 19.
3. Smith CL. When should the stone patient be evaluated? Early evaluation of single stone formers. Med Clin North Am 1984; 68: 455.

4. Wilson DM. Clinical and laboratory approaches for evaluation of neprholithiasis. J Urol 1989; 141: 770.

5. Johnson CM, Wilson DM, O'Fallon WM, et al. Renal stone epidemiology. A 25 year study in Rochester, Minnesota. Kidney Int 1979; 16: 624.

6. Williams RE. Long term survey of 538 patients with upper urinary tract stone. Br J Urol 1963; 35: 416.

7. Strauss AL, Coe FL, Parks JH. Formation of a single calcium stone of renal origin. Clincal and laboratory characteristics in patients. Arch Intern Med 1982; 142: 504.

8. Pak CYC. Should patients with single renal stone occorrence undergo diagnostic evaluation? J Urol 1982; 127: 855 .

9. Pak CYC, Peters P, Hurt G, et al. Is selective therapy of recurrent nephrolithiasis possible. Am J Med 1981; 71: 615 .

10. Hosking D, Erickson SB, van den Berg CJ, et al. The stone clinic effect in patients with idiopathic calcium urolithiasis. J Urol 1983; 130: 1115 .

11. Baggio B, Gambaro G, et al. An inheritable anomaly of red-cell oxalate transport in "primary" calcium nephrolithiasis correctable with diuretics. N Engl J Med 1986; 314: 599.

12. Gambaro G, Marchini F, Vincenti $M$, et al. Clinical features of idiopathic calium-oxalate nephrolithiasis associated with the anomalous erythrocyte self-exchange of oxalate. PRoc. 5th Eur Urolithiasis Symp, Manchester, 1994; 21-23 April: 67. 